\title{
Whole-genome sequencing data of Kazakh individuals
}

\author{
Ulykbek Kairov*0, Askhat Molkenov', Saule Rakhimova², Ulan Kozhamkulov², Aigul Sharip', \\ Daniyar Karabayev' ${ }^{1}$ Asset Daniyarov ${ }^{1}$, Joseph H.Lee ${ }^{3}$, Joseph D.Terwilliger ${ }^{3}$, Ainur Akilzhanova² \\ and Zhaxybay Zhumadilov ${ }^{4}$
}

\begin{abstract}
Objectives: Kazakhstan is a Central Asian crossroad of European and Asian populations situated along the way of the Great Silk Way. The territory of Kazakhstan has historically been inhabited by nomadic tribes and today is the multiethnic country with the dominant Kazakh ethnic group. We sequenced and analyzed the whole-genomes of five ethnic healthy Kazakh individuals with high coverage using next-generation sequencing platform. This whole-genome sequence data of healthy Kazakh individuals can be a valuable reference for biomedical studies investigating disease associations and population-wide genomic studies of ethnically diverse Central Asian region.

Data description: Blood samples have been collected from five ethnic healthy Kazakh individuals living in Kazakhstan. The genomic DNA was extracted from blood and sequenced. Sequencing was performed on Illumina HiSeq2000 next-generation sequencing platform. We sequenced and analyzed the whole-genomes of ethnic Kazakh individuals with the coverage ranging from 26 to 32X. Ranging from 98.85 to $99.58 \%$ base pairs were totally mapped and aligned on the human reference genome GRCh37 hg 19. Het/Hom and Ts/Tv ratios for each whole genome ranged from 1.35 to 1.49 and from 2.07 to 2.08, respectively. Sequencing data are available in the National Center for Biotechnology Information SRA database under the accession number PRJNA374772.
\end{abstract}

Keywords: Whole genome, Next-generation sequencing, Kazakh ethnicity, Bioinformatics analysis, Population genomics, Genome annotation, Biomedical research, Kazakhstan

\section{Objective}

Recent improvements in sequencing technology (nextgeneration and third-generation sequencing platforms) have sharply reduced the cost of sequencing. Nextgeneration sequencing has been developed in order to improve throughput and facilitate the establishment of large-scale data sets in a relatively short time $[1,2]$. The 1000 Genomes Project is an international research effort to sequence a large number of people and establish the most detailed catalogue of human genetic variation [3].

\footnotetext{
*Correspondence: ulykbek.kairov@nu.edu.kz

${ }^{1}$ Laboratory of Bioinformatics and Systems Biology, Center for Life

Sciences, National Laboratory Astana, Nazarbayev University, Nur-Sultan,

Kazakhstan

Full list of author information is available at the end of the article
}

There are currently different sources of complete human genomes publicly available $[4,5]$. Moreover, in most of the countries the national initiatives devoted to wholegenome sequencing, such as $100 \mathrm{~K}$ genomes (England) have been initiated and ongoing.

Kazakhstan is the multi-ethnic and ninth largest country in the world located in Central Asia with the dominant Kazakh ethnic group [6]. Kazakhs have been strongly influenced by the nomadic lifestyle, and a long history of migration has led to admixture of Western and Asian populations, which has formed the genetic background. Thus, it is crucial to understand the genetic architecture of ethnic Kazakhs to properly investigate the genetic basis of common traits in Kazakh population. Despite the research success on comparative population 
studies and whole-genome sequencing of different ethnic groups, Kazakh population genomes remain absent and underrepresented in the majority of available human genome databases.

Here, we report the first complete genome sequences and analysis based on quality metrics of Kazakh ethnic individual's generated using next-generation sequencing platform and available for further investigations. Wholegenome sequencing data obtained at high coverage (26X32X) from four males and one female from Kazakhstan. The resulting whole genome sequences and analysis of Kazakh individuals represent an important and valuable contribution to our knowledge of the genetic landscape of the Central Asian region. Moreover these data may serve as useful resource for application in biomedicine and clinical practice to compare disease specific genetic variants with healthy/normal variants. But considering significant advancement of next-generation technologies and existence of different protocols, it is important to follow the standardized quality management rules for making efficient downstream analysis and utilization of same dataset for different purposes [7].

\section{Data description}

Blood samples from four males and one female have been collected from Kazakh individuals living in Kazakhstan. Genomic DNA was isolated from peripheral blood using Qiagen QIAmp DNA blood mini kit. Concentration of DNA was measured using NanoDrop 2000 spectrophotometer (Thermo Fisher Scientific, USA) and Qubit Fluorimeter 2.0 (Thermo Fisher Scientific, USA). DNA quality was checked using Bioanalyzer 2100 (Agilent Technologies, USA). Paired-end DNA libraries were prepared from $1 \mu \mathrm{g}$ of gDNA using Illumina TruSeq DNA Sample Preparation kit according to manufacturer's protocol (Illumina, USA). Input gDNA amounts have been sheared by Covaris. End repair and $3^{\prime}$ adenylation steps have been performed by End repair mix and A-tailing mix, correspondingly. Indexed paired-end adapters were purified on a gel with following steps of PCR amplification and library validation for fragment size using Agilent HS DNA kit and Qubit HS DNA Assay kit. 300-400 base pairs library insert size has been selected. gDNA fragments were hybridized to the flow cell surface by TruSeq PE Cluster kit v.3 cBot HS and amplified to form clusters using Illumina cBot. High-throughput sequencing was performed by TruSeq SBS kit v.3 HS. Samples sequenced using Illumina HiSeq2000 platform to target 30-fold coverage using paired-end sequencing.

Conversion of generated bcl files to fastq format has been performed using Bcl2fastq tool. We generated 473.4 Gb of data and 4,743,332,930 short reads with average coverage $29 \mathrm{X}$ for five sequenced samples. The quality assessment of raw sequence reads was performed with FastQC v.0.11.7 [8]. Reads were aligned and assembled on the human reference genome (NCBI GRCh37, hg19) and reference mitochondrial DNA rCRS (NC_012920) using Burrows-Wheeler Aligner v.0.7.12 [9]. Alignments corresponding to specific samples were merged into a single BAM file (Data files 1 to 5-Table 1) and marked for duplicates using Picard tools v.1.130. The alignment quality was assessed using SAMtools v.1.2 [10].

From 98.85 to $99.58 \%$ base pairs were totally mapped with properly mapped $99.06 \%$ on average. Het $/ \mathrm{Hom}$ and Ts/Tv ratios for each whole genome ranged from 1.35 to 1.49 and from 2.07 to 2.08 , respectively (Data file 6-Table 1). As a measure of the quality of our whole-genome sequencing data, human genome studies particularly from the 1000 Genomes project have been showing that for whole genomes, a Ts/Tv ratio of around $2-2.1$ is generally a good quality ratio $[3,11$, 12]. Het/Hom ratio also can be used for whole genome sequencing quality metric, but highly depends on ancestry and varies in different populations [13, 14].

Table 1 Overview of data files/data sets

\begin{tabular}{|c|c|c|c|}
\hline Label & Name of data file/data set & $\begin{array}{l}\text { File types } \\
\text { (file extension) }\end{array}$ & Data repository and identifier (DOI or accession number) \\
\hline Data file 1 & SRX2563808/PRJNA374772 & BAM file (.gz) & NCBI SRA https://identifiers.org/ncbi/insdc.sra:SRX2563808 [15] \\
\hline Data file 2 & SRX2563806/PRJNA374772 & BAM file (.gz) & NCBI SRA https://identifiers.org/ncbi/insdc.sra:SRX2563806 [16] \\
\hline Data file 3 & SRX2563805/PRJNA374772 & BAM file (.gz) & NCBI SRA https://identifiers.org/ncbi/insdc.sra:SRX2563805 [17] \\
\hline Data file 4 & SRX2563804/PRJNA374772 & BAM file (.gz) & NCBI SRA https://identifiers.org/ncbi/insdc.sra:SRX2563804 [18] \\
\hline Data file 5 & SRX2563803/PRJNA374772 & BAM file (.gz) & NCBI SRA https://identifiers.org/ncbi/insdc.sra:SRX2563803 [19] \\
\hline Data file 6 & SuppTable_S1-SeqAndMappingSummary.docx & Word file (.docx) & $\begin{array}{l}\text { https://github.com/LabBandSB/wgs_pipeline_on_hg19/raw/master/ } \\
\text { SuppTable_S1-SeqAndMappingSummary.docx } \\
\text { [20] }\end{array}$ \\
\hline Data file 7 & $\begin{array}{l}\text { SuppTable_S2-MappingOf UnmappedRead- } \\
\text { sToNCBlscaffolds.docx }\end{array}$ & Word file (.docx) & $\begin{array}{l}\text { https://github.com/LabBandSB/wgs_pipeline_on_hg19/raw/maste } \\
\text { r/SuppTable_S2-MappingOf\%20UnmappedReadsToNCBIscaffolds } \\
\text {.docx [21] }\end{array}$ \\
\hline
\end{tabular}


Additionally we mapped unmapped sequencing reads to unanchored NCBI human scaffolds and reported that from 411 to 629 unmapped sequencing reads were mapped what corresponds from 0.021 to 0.037 percentage from total number of unmapped sequences (Data file 7-Table 1). The rest of unmapped sequencing reads may suggest for ethnical or individual uniqueness and could be other factors affecting the mapping such as contamination or sequencing errors.

Here we described high-coverage whole genome sequencing data and analysis based on quality metrics of Kazakh individuals representing a valuable resource for the research community complementing the world's genomics map on a global population scale.

\section{Limitations}

Small sample size of described individuals as well as the only next-generation sequencing approach applied without replication performed using the other technologies such genotyping by microarray or third-generation sequencing are limitations of our work.

\begin{abstract}
Abbreviations
DNA: Deoxyribonucleic acid; gDNA: Genomic DNA; Het/Hom: Heterozygous/ Homozygous; Ts/Tv: Transitions/Transversions; PCR: Polymerase chain reaction; NCBI: National Center for Biotechnology Information; SRA: Sequence Read Archive; BAM: Binary alignment map.
\end{abstract}

\section{Acknowledgements}

Not applicable.

\begin{abstract}
Authors' contributions
UK wrote the manuscript; SR, UKo, and AA contributed to experimental works and validation; UK, AM, AS, DK, AD, JDT and JHL contributed to data analysis and implementation of software/code; SR, UKo, AA and ZZ contributed to enrolling patients in the study and provided the clinical data; UK, AA, JHL and ZZ conceptualized the research; UK, AA, and ZZ supervised the research and involved in funding acquisition. All the authors read and approved the final manuscript.
\end{abstract}

\section{Funding}

This work has been supported by grant projects (AP05136106, AP05135430, AP05134722, AP08855353 and AP05134737) from the Committee of Science, Ministry of Education and Science of the Republic of Kazakhstan.

\section{Availability of data and materials}

The data described in this Data note can be freely and openly accessed on NCBI SRA Database under PRJNA374772 accession number. Please see Table 1 and references [15-21] for details and links to the data as well as sequencing and mapping summary and mapping of unmapped reads to NCBI unanchored scaffolds summary.

\section{Ethics approval and consent to participate}

This work was discussed by institutional review board and was approved by the ethical committee of the Center for Life Sciences, National Laboratory Astana, Nazarbayev University (protocol \#3, April 4, 2012). Institutional written informed consent about nationality declaring, DNA extraction, sequencing and for further investigation was signed and obtained from the participated Kazakh individuals.

\section{Consent for publication}

Institutional informed consents were obtained from individuals participated in the investigation.

\section{Competing interests}

The authors declare that they have no competing interests.

\section{Author details}

${ }^{1}$ Laboratory of Bioinformatics and Systems Biology, Center for Life Sciences, National Laboratory Astana, Nazarbayev University, Nur-Sultan, Kazakhstan. ${ }^{2}$ Laboratory of Genomic and Personalized Medicine, Center for Life Sciences, National Laboratory Astana, Nazarbayev University, Nur-Sultan, Kazakhstan.

${ }^{3}$ Columbia University, New York, USA. ${ }^{4}$ School of Medicine, Nazarbayev University, Nur-Sultan, Kazakhstan.

Received: 2 October 2020 Accepted: 28 January 2021

Published online: 04 February 2021

\section{References}

1. Koboldt DC, Steinberg KM, Larson DE, Wilson RK, Mardis ER. The nextgeneration sequencing revolution and its impact on genomics. Cell. 2013;155(1):27-38.

2. Kraft F, Kurth I. Long-read sequencing to understand genome biology and cell function. Int J Biochem Cell Biol. 2020;126:105799.

3. Abecasis GR, Altshuler D, Auton A, Brooks LD, Durbin RM, Gibbs RA, et al. A map of human genome variation from population-scale sequencing. Nature. 2010;467(7319):1061-73.

4. Mallick S, Li H, Lipson M, Mathieson I, Gymrek M, Racimo F, et al. The Simons Genome Diversity Project: 300 genomes from 142 diverse populations. Nature. 2016;538(7624):201-6.

5. Bergstrom A, McCarthy SA, Hui R, Almarri MA, Ayub Q, Danecek P, et al. Insights into human genetic variation and population history from 929 diverse genomes. Science. 2020;367(6484):eaay5012.

6. The republic of Kazakhstan. https://www.akorda.kz/en/republic_of_kazak hstan/kazakhstan. Accessed 02 Oct 2020.

7. Endrullat Ch, Glökler J, Franke Ph, Frohme M. Standardization and quality management in next-generation sequencing. Appl Transl Genomics. 2016;1(10):2-9.

8. FastQC tool. http://www.bioinformatics.bbsrc.ac.uk/projects/fastqc.

9. Li H, Durbin R. Fast and accurate long-read alignment with BurrowsWheeler transform. Bioinformatics. 2010;26(5):589-95.

10. Li H, Handsaker B, Wysoker A, Fennell T, Ruan J, Homer N, et al. The Sequence Alignment/Map format and SAMtools. Bioinformatics. 2009;25(16):2078-9.

11. Guo Y, Ye F, Sheng QH, Clark T, Samuels DC. Three-stage quality control strategies for DNA re-sequencing data. Brief Bioinform. 2014;15(6):879-89.

12. Wang J, Raskin L, Samuels DC, Shyr Y, Guo Y. Genome measures used for quality control are dependent on gene function and ancestry. Bioinformatics. 2015;31(3):318-23.

13. Tuzov N. A framework for the estimation of the proportion of true discoveries in single nucleotide variant detection studies for human data. PLoS ONE. 2018;13(4):e0196058.

14. Zhu Q, Hu Q, Shepherd L, Wang J, Wei L, Morrison CD, et al. The impact of DNA input amount and DNA source on the performance of wholeexome sequencing in cancer epidemiology. Cancer Epidemiol Biomarkers Prev. 2015:24(8):1207-13.

15. National Center for Biotechnology Information SRA database; 2020. https ://www.ncbi.nlm.nih.gov/sra/SRX2563808.

16. National Center for Biotechnology Information SRA database; 2020. https ://www.ncbi.nlm.nih.gov/sra/SRX2563806.

17. National Center for Biotechnology Information SRA database; 2020. https ://www.ncbi.nlm.nih.gov/sra/SRX2563805.

18. National Center for Biotechnology Information SRA database; 2020. https ://www.ncbi.nlm.nih.gov/sra/SRX2563804.

19. National Center for Biotechnology Information SRA database; 2020. https ://www.ncbi.nlm.nih.gov/sra/SRX2563803. 
20. Sequencing and mapping summary; 2020. https://github.com/LabBa ndSB/wgs_pipeline_on_hg19/raw/master/SuppTable_S1-SeqAndMapp ingSummary.docx.

21. Mapping of unmapped reads to NCBI scaffolds; 2020. https://githu b.com/LabBandSB/wgs_pipeline_on_hg19/raw/master/SuppTable_ S2-MappingOf\%20UnmappedReadsToNCBlscaffolds.docx.

\section{Publisher's Note}

Springer Nature remains neutral with regard to jurisdictional claims in published maps and institutional affiliations.
Ready to submit your research? Choose BMC and benefit from:

- fast, convenient online submission

- thorough peer review by experienced researchers in your field

- rapid publication on acceptance

- support for research data, including large and complex data types

- gold Open Access which fosters wider collaboration and increased citations

- maximum visibility for your research: over 100M website views per year

At BMC, research is always in progress.

Learn more biomedcentral.com/submissions 